\title{
Analysis of cardiac autonomic modulation of children with attention deficit hyperactivity disorder
}

This article was published in the following Dove Press journal:

Neuropsychiatric Disease and Treatment

I I April 2014

Number of times this article has been viewed

\author{
Tatiana Dias de Carvalho ${ }^{1,2}$ \\ Rubens Wajnsztejn ${ }^{3}$ \\ Luiz Carlos de Abreu ${ }^{2,7}$ \\ Luiz Carlos Marques \\ Vanderlei ${ }^{4}$ \\ Moacir Fernandes Godoy ${ }^{5}$ \\ Fernando Adami \\ Vitor E Valenti ${ }^{6}$ \\ Carlos B M Monteiro ${ }^{2,7}$ \\ Claudio Leone ${ }^{7}$ \\ Karen Cristina da Cruz \\ Martins ${ }^{2}$ \\ Celso Ferreira'
}

'Departamento de Medicina, Disciplina de Cardiologia, Universidade Federal de São Paulo, UNIFESP, São Paulo, Brazil; ${ }^{2}$ Laboratório de Escrita Científica da Faculdade de Medicina do ABC, FMABC, Santo André, Brazil; ${ }^{3}$ Núcleo Especializado em Aprendizagem, Programa de pósgraduação em Ciências da Saúde da Faculdade de Medicina do ABC, FMABC, Santo André, Brazil; ${ }^{4}$ Departamento de Fisioterapia da Faculdade de Ciências e Tecnologia, Universidade Estadual Paulista, UNESP, Presidente Prudente, São Paulo, Brazil; ${ }^{5}$ Núcleo Transdisciplinar de Estudos do Caos e da Complexidade. Faculdade de Medicina de São José de Rio Preto, FAMERP, São José do Rio Preto, Brazil; 'Departamento de Fonoaudiologia da Faculdade de Filosofia e Ciências, Universidade Estadual Paulista, UNESP, Marília, Brazil; ' ${ }^{7}$ epartamento de Saúde Materno-Infantil da Faculdade de Saúde Pública da Universidade de São Paulo, São Paulo, Brazil

Correspondence: Luiz Carlos de Abreu Faculdade de Medicina do ABC.

Av. Príncipe de Gales, 82।

Príncipe de Gales - Santo André/SP - CEP 09060, Brazil

Email luiz.abreu@fmabc.br
Background: Attention deficit hyperactivity disorder (ADHD) is characterized by decreased attention span, impulsiveness, and hyperactivity. Autonomic nervous system imbalance was previously described in this population. We aim to compare the autonomic function of children with ADHD and controls by analyzing heart rate variability (HRV).

Methods: Children rested in supine position with spontaneous breathing for 20 minutes. Heart rate was recorded beat by beat. HRV analysis was performed in the time and frequency domains and Poincaré plot.

Results: Twenty-eight children with ADHD (22 boys, aged 9.964 years) and 28 controls ( 15 boys, age 9.857 years) participated in this study. It was determined that the mean and standard deviation of indexes which indicate parasympathetic activity is higher in children with ADHD than in children without the disorder: high frequency in normalized units, 46.182 (14.159) versus 40.632 (12.247); root mean square of successive differences, 41.821 (17.834) versus 38.150 (18.357); differences between adjacent normal-to-normal intervals greater than 50 milliseconds, 199.75 (144.00) versus 127.46 (102.21) $(P<0.05)$; percentage of differences between adjacent normal-to-normal intervals greater than 50 milliseconds, 23.957 (17.316) versus 16.211 (13.215); standard deviation of instantaneous beat-to-beat interval, 29.586 (12.622) versus 26.989 (12.983).

Conclusion: Comparison of the autonomic function by analyzing HRV suggests an increase in the activity of the parasympathetic autonomic nervous systems in children with ADHD in relation to the control group.

Keywords: attention deficit hyperactivity disorder, autonomic nervous system, children, heart rate variability

\section{Introduction}

Attention deficit hyperactivity disorder (ADHD) is characterized by decreased attention span, impulsiveness, and hyperactivity. ${ }^{1,2}$ It is a neurobiological condition with a genetic basis, ${ }^{3}$ with a prevalence of $3 \%$ to $7 \%$ of the population, including children, adolescents, and adults. The incidence is higher in men. ${ }^{4,5}$

There is evidence that ADHD is more prevalent in children with cardiac diseases ${ }^{4}$ and drug treatment has effects on cardiovascular parameters, such as heart rate and blood pressure. ${ }^{6-9}$

Considering that cardiac function is regulated by the interaction of sympathetic and parasympathetic branches of the autonomic nervous system (ANS), heart rate variability (HRV) allows the investigation of fluctuations in the intervals between consecutive heart beats (RR intervals), which are related to the influences of the ANS on the sinus node. ${ }^{10}$ This is a noninvasive technique, which may be performed by using 
linear methods, time and frequency domain, ${ }^{11}$ and nonlinear methods such as Poincaré plot. ${ }^{10-12}$

Alterations in autonomic activity in children with ADHD have been investigated in several studies. ${ }^{6,8,13-15}$ Negrao et al $^{16}$ found that stimulant-free children with ADHD have a parasympathetic dominance of the autonomic balance relative to control subjects. However, Crowell et $\mathrm{al}^{17}$ indicated that stimulant-free children with ADHD do not differ from age-matched controls, with regard to parasympathetic nervous system activity. Other studies suggest that children with ADHD display underactivity of the parasympathetic nervous system when compared to controls. ${ }^{17,18}$

It is known that changes in cardiac autonomic modulation, reflected by reduced HRV, have been identified as an indicator of poor prognosis in cardiovascular diseases, ${ }^{19,20}$ and it is also related to the development of arrhythmia ${ }^{21}$ and sudden death. ${ }^{22}$

We hypothesized that children with ADHD present alterations in autonomic activity. Thus, the aim of this study was to compare the autonomic function of children with ADHD and controls by analyzing HRV.

\section{Materials and methods Population}

All patients were recruited from the Núcleo Especializado em Aprendizagem da Faculdade de Medicina do ABC (Learning Disabilities Center of Medicine ABC University), Santo André, Brazil, where they are treated by a multidisciplinary team. All volunteers in this study had the medical diagnosis of ADHD, according to the Diagnostic and Statistical Manual of Mental Disorders, 4th edition, published by the American Psychological Association, 1,2 and all met the following inclusion criteria: 1) absence of congenital anomalies such as congenital heart disease, pulmonary malformations, diaphragmatic hernia, and defects of abdominal wall closure; 2) absence of central nervous system malformations and/or neurological syndromes; 3 ) absence of metabolic disorders; 4) absence of medication that influences cardiac autonomic modulation since that drug treatment (eg, methylphinedite) has effects on cardiovascular parameters such as heart rate and blood pressure. The control group was composed of healthy children without a diagnosis of ADHD.

Parents of the children were asked for consent. The study received approval from the Ethics Committee of Faculdade de Medicina do ABC (protocol number 312/11) and was in compliance with Resolution 196/96 of the Conselho Nacional de Saúde.

\section{Procedure}

Data were collected under controlled temperature $\left(21^{\circ} \mathrm{C}-23^{\circ} \mathrm{C}\right)$ and humidity $(40 \%-60 \%)$. Participants went to the laboratory between 8 am and $11 \mathrm{am}$. They were instructed to avoid consuming caffeine for 24 hours before evaluation. Parents and guardians of the children stayed in the room during the entire protocol.

Demographics and anthropometric measurements were obtained following the standard recommendations proposed by Lohman et al. ${ }^{23}$ A heart monitor strap was placed on the chest, over the distal third of the sternum, and the heart rate receiver (Polar RS800 CX monitor; Polar, Kempele, Finland) was placed on the wrist. This type of equipment has been previously validated for beat-by-beat measurements and for HRV analysis. ${ }^{24-26}$ Children rested in supine position with spontaneous breathing for 20 minutes and were instructed to avoid talking during data collection.

\section{Analysis of heart rate variability}

For HRV analysis, the heart rate was recorded beat-by-beat at a sampling frequency of $1,000 \mathrm{~Hz}$. The data series was first digitally filtered using Polar Precision Performance SW software (version 4.01.029; Polar), in which only series with more than $95 \%$ sinus rhythm beats were included. ${ }^{19}$ It was then manually complemented, and the visual inspection of the time series on the computer showed absence of artifacts. Finally, 1,000 RR intervals were selected for data analysis. HRV analysis was performed with linear methods in the domains of time and frequency. ${ }^{10,11}$

In the time domain, we used the root mean square of successive differences (RMSSD), the percentage of differences between adjacent normal-to-normal intervals greater than 50 milliseconds ( $p N N 50$ ), and the differences between adjacent normal-to-normal intervals greater than 50 milliseconds (NN50) to indicate isolated parasympathetic activity, and the standard deviation of normal-to-normal intervals (SDNN) represented global variability. ${ }^{10,11}$

In the frequency domain, the spectral components of low frequency (LF) (0.04-0.15 Hz), which indicates global variability, and high frequency (HF) $(0.15-0.40 \mathrm{~Hz})$, which indicates parasympathetic activity in normalized units (LFnu and HFnu, respectively) and in milliseconds squared were used, as well as the ratio between these components (LF/HF). Spectral analysis was performed using the fast Fourier transform algorithm. ${ }^{10,11}$

The Poincaré plot was also analyzed. This plot is a graphic representation of the two-dimensional correlation 
between RR intervals, where each interval is plotted against the next interval. ${ }^{12,27}$ This quantitative analysis is performed by fitting the ellipse of the figure formed by the attractor from where the indexes are obtained: SD1, SD2 and SD1/ SD2 ratio. The SD1 index means standard deviation of instantaneous beat-to-beat RR interval variability and represents vagal modulation. The SD2 index means the standard deviation of continuous long-term RR interval variability and represents global variability. ${ }^{10,11}$ The SD1/SD2 ratio indicates the relationship between parasympathetic and overall HRV. ${ }^{27,28}$

For analysis of indexes of HRV, we used the software program Kubios HRV (Biosignal Analysis and Medical Image Group, University of Eastern Finland, Kuopio, Finland). ${ }^{29}$

\section{Statistical analysis}

Descriptive statistics with estimates of mean and standard deviation were used. The normality of the data was determined using the Shapiro-Wilk test. Comparisons between groups were performed using either the independent Student's $t$-test or Mann-Whitney $U$-test for parametric (age, SDNN, NN50, pNN50, LFnu, HFnu, SD2, and SD1/SD2) or for nonparametric (weight, height, body mass index [BMI], RR interval, RMSSD, LF milliseconds ${ }^{2}$, HF milliseconds ${ }^{2}$, LF/HF, and SD1) sample distribution. Differences were considered significant when the probability of a type I error was lower than $5 \%(P<0.05)$. The statistical program InStat 3 (GraphPad Software Inc., La Jolla, CA, USA) was used.

\section{Results}

We selected 44 children with ADHD, of whom 16 were excluded for not meeting the following criteria: series with less than $95 \%$ sinus rhythm beats and any medication that influences the cardiac autonomic modulation. Twenty-eight children participated in this study ( 22 boys and six girls, aged 9.964 years). The control group comprised 28 children ( 15 boys and 13 girls, age 9.857 years) without ADHD.

Table 1 shows average values for age and anthropometric characteristics of ADHD children and controls. Table 2 displays the values obtained for LF and HF indexes in normalized units and in milliseconds ${ }^{2}$ and the LF/HF ratio in children with ADHD and controls. Table 3 depicts the values for the RR interval and the SDNN, RMSSD, NN50, and pNN50 indexes. Table 4 displays the values of the SD1 and $\mathrm{SD} 2$ indexes and the SD1/SD2 ratio in children with $\mathrm{ADHD}$ and controls.
Table I Average values and standard deviations for age, weight, height, and body mass index in ADHD children and controls

\begin{tabular}{llll}
\hline Variables & ADHD & Controls & $P$ \\
& Average (SD) $(\mathrm{Cl})$ & Average (SD) $(\mathrm{Cl})$ & \\
\hline Age (years) & $9.96(\mathrm{I} .93)$ & $9.85(\mathrm{I}) 84)$ & 0.832 \\
& $(9.2 \mathrm{I}-10.7 \mathrm{I})$ & $(9.14-10.57)$ & \\
Weight $(\mathrm{kg})$ & $38.76(17.83)$ & $35.07(9.09)$ & 0.682 \\
& $(31.84-45.67)$ & $(31.55-38.60)$ & \\
Height (m) & $1.39(0.13)$ & $1.44(0.10)$ & 0.060 \\
& $(1.34-1.44)$ & $(1.40-1.48)$ & \\
BMI $\left(\mathrm{kg} / \mathrm{m}^{2}\right)$ & $19.20(4.67)$ & $16.52(1.91)$ & $0.02 I^{*}$ \\
& $(17.39-21.02)$ & $(15.78-17.27)$ & \\
\hline
\end{tabular}

Note: $* P<0.05$.

Abbreviations: ADHD, attention deficit hyperactivity disorder; BMI, body mass index; $\mathrm{Cl}$, confidence interval; SD, standard deviation.

Table 2 Linear index values of heart rate variability (frequency domain) of children with ADHD and controls

\begin{tabular}{|c|c|c|c|}
\hline Variables & $\begin{array}{l}\text { ADHD } \\
\text { Average (SD) (Cl) }\end{array}$ & $\begin{array}{l}\text { Controls } \\
\text { Average (SD) (Cl) }\end{array}$ & $P$ \\
\hline LF $\mathrm{ms}^{2}$ & $\begin{array}{l}781.00(449.07) \\
(606.86-955.14)\end{array}$ & $\begin{array}{l}1021.4(723.95) \\
(740.62-1302.1)\end{array}$ & 0.305 \\
\hline $\mathrm{HF} \mathrm{ms}^{2}$ & $\begin{array}{l}751.64(518.60) \\
(550.54-952.75)\end{array}$ & $\begin{array}{l}799.86(828.35) \\
(478.63-1|2| . \mid)\end{array}$ & 0.588 \\
\hline LFnu & $\begin{array}{l}53.8 I(14.15) \\
(48.32-59.30)\end{array}$ & $\begin{array}{l}59.36(12.24) \\
(54.6 I-64.11)\end{array}$ & 0.122 \\
\hline HFnu & $\begin{array}{l}46.18(14.15) \\
(40.69-51.67)\end{array}$ & $\begin{array}{l}40.63(12.24) \\
(35.88-45.38)\end{array}$ & 0.122 \\
\hline $\mathrm{LF} / \mathrm{HF}$ & $\begin{array}{l}1.37(0.75) \\
(1.08-1.66)\end{array}$ & $\begin{array}{l}1.74(1.05) \\
(1.33-2.14)\end{array}$ & 0.187 \\
\hline
\end{tabular}

Abbreviations: $A D H D$, attention deficit hyperactivity disorder; $\mathrm{Cl}$, confidence interval; HF, high frequency; HFnu, high frequency in normalized units; LF, low frequency; LFnu, low frequency in normalized units; ms, milliseconds; SD, standard deviation.

Table 3 Linear index values of heart rate variability (time domain) of children with ADHD and controls

\begin{tabular}{|c|c|c|c|}
\hline Variables & $\begin{array}{l}\text { ADHD } \\
\text { Average (SD) (Cl) }\end{array}$ & $\begin{array}{l}\text { Controls } \\
\text { Average (SD) (Cl) }\end{array}$ & $P$ \\
\hline $\mathrm{RRi}$ & $\begin{array}{l}714.46(84.15) \\
(681.83-747.10)\end{array}$ & $\begin{array}{l}680.86(99.23) \\
(642.38-719.35)\end{array}$ & 0.108 \\
\hline SDNN & $\begin{array}{l}48.56(13.34) \\
(43.39-53.74)\end{array}$ & $\begin{array}{l}54.5 I(18.60) \\
(47.29-61.72)\end{array}$ & 0.175 \\
\hline RMSSD & $\begin{array}{l}41.82(17.83) \\
(34.90-48.73)\end{array}$ & $\begin{array}{l}38.15(18.35) \\
(31.03-45.26)\end{array}$ & $0.34 I$ \\
\hline NN50 & $\begin{array}{l}199.75(144.00) \\
(143.91-255.59)\end{array}$ & $\begin{array}{l}127.46(102.21) \\
(87.82-167.10)\end{array}$ & $0.035 *$ \\
\hline pNN50 & $\begin{array}{l}23.95(17.31) \\
(17.24-30.67)\end{array}$ & $\begin{array}{l}|6.2|(|3.2|) \\
(|1.08-2| .33)\end{array}$ & 0.065 \\
\hline
\end{tabular}

Note: $* p<0.05$.

Abbreviations: ADHD, attention deficit hyperactivity disorder; $\mathrm{Cl}$, confidence interval; NN50, adjacent normal-to-normal intervals that are greater than $50 \mathrm{~ms}$; pNN50, percentage of differences between adjacent normal-to-normal intervals that are greater than $50 \mathrm{~ms}$; ms, milliseconds; RMSSD, root mean square of successive differences; RRi, intervals between consecutive heart beats; SD, standard deviation; SDNN, standard deviation normal-to-normal intervals. 
Table 4 Mean, standard deviation, and 95\% confidence interval for Poincaré plot of children with ADHD and controls

\begin{tabular}{llll}
\hline Variables & $\begin{array}{l}\text { ADHD } \\
\text { Average (SD) }(\mathrm{Cl})\end{array}$ & $\begin{array}{l}\text { Controls } \\
\text { Average (SD) }(\mathrm{Cl})\end{array}$ & $\mathbf{P}$ \\
\hline SDI & $29.58(12.62)$ & $26.98(12.98)$ & 0.333 \\
& $(24.69-34.48)$ & $(21.95-32.02)$ & \\
SD2 & $61.64(15.68)$ & $71.94(23.66)$ & $0.06 \mathrm{I}$ \\
& $(55.56-67.72)$ & $(62.76-81.12)$ & \\
SDI/SD2 & $0.46(0.12)$ & $0.36(0.095)$ & $0.002^{*}$ \\
& $(0.41-0.5 \mathrm{I})$ & $(0.33-0.40)$ & \\
\hline
\end{tabular}

Note: $* P<0.05$.

Abbreviations: ADHD, attention deficit hyperactivity disorder; $\mathrm{Cl}$, confidence interval; SD, standard deviation; SDI, standard deviation of instantaneous beat-tobeat RR interval variability; SD2, standard deviation of continuous long-term RR interval variability.

\section{Discussion}

ADHD is one of the most commonly diagnosed mental disorders among children. With regard to anthropometric characteristics of the studied groups, as presented in Table 1, there was no significant difference in age, weight, and height. BMI values were higher in children with ADHD than those without the disorder.

Although obesity causes alterations in cardiac autonomic function, ${ }^{27,30}$ the observed difference in BMI values did not influence our results with respect to HRV.

Contrary to findings in the literature which describe decreased activity of the parasympathetic ANS in obese children, ${ }^{28,31}$ our evaluation of the HRV indexes in the present study suggests that despite higher BMI, children with ADHD had an increase in activity of the parasympathetic ANS in relation to the control group.

In this investigation, according to the frequency and time domains and the Poincaré plot, the indexes that indicate parasympathetic activity were higher in children with ADHD than in children without the disorder.

Alterations in autonomic activity in children with ADHD have been investigated in several studies, ${ }^{6,8,13-15}$ and an increase in parasympathetic activity among these children has also been reported. ${ }^{16}$ In a study that evaluated ANS functioning and the effects of methylphenidate in stimulantfree children with ADHD and controls, Negrao et a ${ }^{16}$ found, by analyzing the time and frequency domains and Poincaré plot indexes, that stimulant-free children with ADHD have a parasympathetic dominance of the autonomic balance relative to control subjects.

Their results are consistent with our findings since the results of both frequency and time domains in our study suggest an increase in parasympathetic activity in children with ADHD. The indexes HFnu (Table 2), RMSSD, NN50,
pNN50 (Table 3), and SD1 (Table 4) were higher in children with ADHD than in children without the disorder. In the time domain, we found a significant difference $(P=0.035)$ in the NN50 index, indicating isolated parasympathetic activity. ${ }^{10,11}$

Nevertheless, our results do not support the parasympathetic nervous system findings from Crowell et al, ${ }^{17}$ which indicated that children with ADHD without stimulant do not differ from age-matched controls with regard to parasympathetic activity, nor the findings of Shibagaki and Furuya, ${ }^{18}$ which suggest that children with ADHD display an underactivity of the parasympathetic nervous system when compared to controls. Both studies also evaluated the respiratory sinus arrhythmia and heart rate responses. ${ }^{17,18}$

The reasons for the similar or different findings may be due to variation in methods of research. The other studies differed in protocol (autonomic tests, focused attention, auditory stimulation), population (included children with oppositional defiant disorder and different age), and assessed indexes (only one study evaluated Poincaré plot).

Parasympathetic nervous system-linked cardiac activity has been associated with emotion regulation capabilities. ${ }^{14,15}$ Porges Polyvagal Theory, ${ }^{31}$ for example, posits that the myelinated vagus, originating in the nucleus ambiguus, is a dynamic contributor to the processes of attention, motion, emotion, and social interactions. The theory has introduced a new perspective, relating autonomic function to behavior, but it requires further study for a better understanding of communication between the heart and the central nervous system. ${ }^{13,31}$

With respect to the indexes which indicate global variability and sympathetic activity (LF in the frequency domain and SDNN in the time domain), we found no statistically significant differences; however, these values were lower in children with ADHD compared with control children.

Concerning the Poincaré plot analysis, we observed no statistically significant differences between the SD1 and SD2 indexes, which represent vagal modulation and global variability, respectively. ${ }^{27,28}$ However the SD2 value was lower in children with ADHD compared with control children, indicating a level of sympathetic underarousal in these subjects as suggested by Negrao et al, ${ }^{16}$ who also found lower SD2 values in ADHD children compared with controls.

Also regarding the Poincaré plot analysis, our results showed significant difference $(P=0.0025)$ in the SD1/SD2 ratio, which can be explained by higher SD1 value and lower SD2 value in the ADHD group as compared with controls, who exhibited lower SD1 value and higher SD2 value (Table 4). 
Studies of ANS activity in these children have presented conflicting results. Tonhajzerova et al, ${ }^{13}$ using short-term HRV analysis to evaluate the cardiac autonomic regulation in children with ADHD at rest and during orthostasis, found that the mean RR interval was significantly shorter in the ADHD group compared to controls, in all positions. The authors concluded that children with ADHD had decreased cardiac vagal modulation at rest and during orthostasis, and tachycardia in supine positions, with altered ability of dynamic activation of the ANS in response to orthostasis, indicating changes in the cardiac autonomic regulation. ${ }^{13}$

There have been studies to assess the effect of medication on autonomic modulation and cardiovascular parameters in these individuals. ${ }^{6,15,16}$ In a study analyzing HRV in 24-hour electrocardiogram recordings while under medication with stimulants, Buchhorn et $\mathrm{al}^{15}$ have shown alterations in basal autonomic activity in children with ADHD, with a shift towards normal levels by medication with methylphenidate.

The effect of this medication has also been evaluated in other studies. ${ }^{6,16}$ According to Negrao et al, ${ }^{16}$ methylphenidate acts to restore the normal autonomic balance in children with ADHD, but inhibits the normal ANS response to a cognitive challenge. In another study that investigated the effects of sympathomimetic medication on the cardiovascular system of children with ADHD, statistically significant differences were found in both systolic and diastolic blood pressure. Also, heart rate was higher in children with ADHD while on stimulant medication. The authors concluded that methylphenidate usage is associated with an increase in heart rate and increases in both systolic and diastolic blood pressure. ${ }^{6}$

Our findings were not influenced by medication because absence of medications that influences cardiac autonomic modulation was an inclusion criterion. Some limitations of the current research must be considered. First, there were more boys in the sample with ADHD than the control group. In fact, the prevalence of ADHD is higher in boys than in girls, ${ }^{1,2}$ however, the average values and standard deviations for age did not present significant differences, which leads us to believe that our results were not influenced by hormonal changes in the participants. Second, the physical activity of the groups was not investigated. It is known that the level of physical activity can influence autonomic behavior, increasing the indexes which represent vagal modulation. ${ }^{32}$ We can say that all children participating in this study, with and without ADHD, perform physical activity in their schools as part of the curriculum. Thus, health is a sustainable state ${ }^{33}$ that may be in constant flux and is dependent on constant attention, active maintenance, and care.

In conclusion, the comparison of autonomic function by analyzing HRV suggests an increase in the activity of the parasympathetic ANS in children with ADHD in relation to the control group.

\section{Acknowledgments}

This manuscript received financial support from FAPESP Process 2013/17628-2.

\section{Author contributions}

TDDC and RW contributed equally in the design and data collection of research. All the authors contributed toward data analysis, drafting of the manuscript and agree to be accountable for all aspects of the work.

\section{Disclosure}

The authors report no conflicts of interest in this work.

\section{References}

1. Park S, Kim JW, Yang YH, et al. Possible effect of norepinephrine transporter polymorphisms on methylphenidate-induced changes in neuropsychological function in attention-deficit hyperactivity disorder. Behav Brain Funct. 2012;8:22.

2. Cantwell, DP. Attention deficit disorder: a review of the past 10 years J Am Acad Child Adolesc Psychiatry. 1996;35(8):978-986.

3. Russell VA, Oades RD, Tannock R, et al. Response variability in attention-deficit/hyperactivity disorder: a neuronal and glial energetics hypothesis. Behav Brain Funct. 2006;2:30.

4. Rohde LA, Szobot C, Polanczyk G, Schmitz M, Martins S, Tramontina S. Attention-deficit/hyperactivity disorder in a diverse culture: do research and clinical findings support the notion of a cultural construct for the disorder? Biol Psychiatry. 2005;57(11):1436-1441.

5. Nikolas M, Friderici K, Waldman I, Jernigan K, Nigg JT. Gene x environment interactions for ADHD: synergistic effect of 5HTTLPR genotype and youth appraisals of inter-parental conflict. Behav Brain Funct. 2010;6:23.

6. Negrao BL, Crafford D, Viljoen M. The effect of sympathomimetic medication on cardiovascular functioning of children with attention-deficit/hyperactivity disorder. Cardiovasc J Afr. 2009;20(5): 296-299.

7. Meijer WM, Faber A, van den Ban E, Tobi H. Current issues around the pharmacotherapy of ADHD in children and adults. Pharm World Sci. 2009;31(5):509-516.

8. Ballard JE, Boileau RA, Sleator EK, Massey BH, Sprague RL. Cardiovascular responses of hyperactive children to methylphenidate. JAMA. 1976;236(25):2870-2874.

9. Thayer JF, Lane RD. The role of vagal function in the risk for cardiovascular disease and mortality. Biol Psychol. 2007;74(2): 224-242.

10. Heart rate variability: standards of measurement, physiological interpretation and clinical use. Task Force of the European Society of Cardiology and the North American Society of Pacing and Electrophysiology. Circulation. 1996;93(5):1043-1065.

11. Vanderlei LC, Pastre CM, Hoshi RA, Carvalho TD, Godoy MF. Basic notions of heart rate variability and its clinical applicability. Rev Bras Cir Cardiovasc. 2009;24(2):205-217. 
12. Dias de Carvalho T, Marcelo Pastre C, Claudino Rossi R, de Abreu LC, Valenti VE, Marques Vanderlei LC. [Geometric index of heart rate variability in chronic obstructive pulmonary disease]. Rev Port Pneumol. 2011;17(6):260-265. Portuguese.

13. Tonhajzerova I, Ondrejka I, Adamik P, et al. Changes in the cardiac autonomic regulation in children with attention deficit hyperactivity disorder (ADHD). Indian J Med Res. 2009;130(1):44-50.

14. van Lang ND, Tulen JH, Kallen VL, Rosbergen B, Dieleman G, Ferdinand RF. Autonomic reactivity in clinically referred children attentiondeficit/hyperactivity disorder versus anxiety disorder. Eur Child Adolesc Psychiatry. 2007;16(2):71-78.

15. Buchhorn R, ConzelmannA, Willaschek C, Störk D, Taurines R, RennerTJ. Heart rate variability and methylphenidate in children with ADHD. Atten Defic Hyperact Disord. 2012;4(2):85-91.

16. Negrao BL, Bipath P, van der Westhuizen D, Viljoen M. Autonomic correlates at rest and during evoked attention in children with attention-deficit/hyperactivity disorder and effects of methylphenidate. Neuropsychobiology. 2011;63(2):82-91.

17. Crowell SE, Beauchaine TP, Gatzke-Kopp L, Sylvers P, Mead H, Chipman-Chacon J. Autonomic correlates of attention-deficit/ hyperactivity disorder and oppositional defiant disorder in preschool children. J Abnorm Psychol. 2006;115(1):174-178.

18. Shibagaki M, Furuya T. Baseline respiratory sinus arrhythmia and heartrate responses during auditory stimulation of children with attention-deficit hyperactivity disorder. Percept Mot Skills. 1997;84(3 Pt 1):967-975.

19. Godoy MF, Takakura IT, Correa PR. Relevância da análise do comportamento dinâmico não-linear (Teoria do Caos) como elemento prognóstico de morbidade e mortalidade em pacientes submetidos à cirurgia de revascularização miocárdica. [The relevance of nonlinear dynamic analysis (Chaos Theory) to predict morbidity and mortality in patients undergoing surgical myocardial revascularization]. Arq Ciênc Saúde. 2005;12(4):167-171. Portuguese.

20. Rocha RM, Albuquerque DC, Albanesi Filho FM. Variabilidade da frequência cardíaca e ritmo circadiano em pacientes com angina estável. [Heart rate variability and circadian rhythm in patients with stable angina]. Rev Socerj. 2005;18(5):429-442. Portuguese.

21. Tükek T, Yildiz P, Atilgan D, et al. Effect of diurnal variability of heart rate on development of arrhythmia in patients with chronic obstructive pulmonary disease. Int J Cardiol. 2003;88(2-3):199-206.
22. Hayano J, Takahashi H, Toriyama T, et al. Prognostic value of heart rate variability during long-term follow-up in chronic haemodialysis patients with end-stage renal disease. Nephrol Dial Transplant. 1999;14(6): 1480-1488.

23. Lohman TG, Roche AF, Martorell R, editors. Anthropometric Standardization Reference Manual. Champaign, IL: Human Kinetics; 1988.

24. Gamelin FX, Berthoin S, Bosquet L. Validity of the polar S810 heart rate monitor to measure R-R intervals at rest. Med Sci Sports Exerc. 2006;38(5):887-893.

25. Kingsley M, Lewis MJ, Marson RE. Comparison of Polar 810s and an ambulatory ECG system for RR interval measurement during progressive exercise. Int J Sports Med. 2005;26(1):39-44.

26. Vanderlei LC, Silva RA, Pastre CM, Azevedo FM, Godoy MF. Comparison of the Polar S810i monitor and the ECG for the analysis of heart rate variability in the time and frequency domains. Braz J Med Biol Res. 2008;41(10):854-859.

27. Vanderlei LC, Pastre CM, Freitas Jr IF, Godoy MF. Geometric indexes of heart rate variability in obese and eutrophic children. Arq Bras Cardiol. 2010;95(1):35-40.

28. Vanderlei FM, Rossi RC, Souza NM, et al. Heart rate variability in healthy adolescents at rest. Journal of Human Growth and Development. 2012;22(2):173-178.

29. Niskanen JP, Tarvainen MP, Ranta-Aho PO, Karjalainen PA. Software for advanced HRV analysis. Comput Methods Programs Biomed. 2004;76(1):73-81.

30. Vanderlei LC, Pastre CM, Freitas Júnior IF, Godoy MF. Analysis of cardiac autonomic modulation in obese and eutrophic children. Clinics (Sao Paulo). 2010;65(8):789-792.

31. Porges SW. The polyvagal perspective. Biol Psychol. 2007;74(2): 116-143.

32. Melo RC, Santos MD, Silva E, et al. Effects of age and physical activity on the autonomic control of heart rate in healthy men. Braz J Med Biol Res. 2005;38(9):1331-1338.

33. Atrash HK, Carpentier R. The evolving role of public health in the delivery of health care. Journal of Human Growth and Development. 2012;22(3):396-399.
Neuropsychiatric Disease and Treatment

\section{Publish your work in this journal}

Neuropsychiatric Disease and Treatment is an international, peerreviewed journal of clinical therapeutics and pharmacology focusing on concise rapid reporting of clinical or pre-clinical studies on a range of neuropsychiatric and neurological disorders. This journal is indexed on PubMed Central, the 'PsycINFO' database and CAS.

\section{Dovepress}

The manuscript management system is completely online and includes a very quick and fair peer-review system, which is all easy to use. Visit http://www.dovepress.com/testimonials.php to read real quotes from published authors. 